\title{
Graphical approach to multi-slit interference analysis
}

\author{
Patricia E. Allen
}

\author{
Department of Physics \& Astronomy, Appalachian State University, 525 Rivers St, Boone, NC, 28608
}

\begin{abstract}
When Young's double-slit experiment is conducted in undergraduate lab courses, wavelength or slit spacing is commonly calculated using Young's relation. For multi-slit gratings of $500 \mathrm{lines} / \mathrm{mm}$, or more, few orders of interference fringes are observed, generating inaccurate and imprecise results. Here the author suggests alternate data acquisition and analysis techniques. By graphing average distance $x_{\text {avg }}$ from the $0^{\text {th }}$ order to $1^{\text {st }}$ order fringe as a function of distance from grating to screen $L$ a linear relationship can be obtained, with the slope related to wavelength/slit spacing. With some algebra, accurate (within 1\%) and precise results can be obtained. The experimental set-up used at Appalachian State University will be presented, along with sample student results, including error propagation calculations utilized during a sophomore/junior lab "intermediate" lab course (precursor to capstone lab experience).
\end{abstract}

\section{INTRODUCTION}

Young's double-slit experiment is often utilized in physics laboratory courses to demonstrate the wave property of light. By extension, interference patterns from multi-slit diffraction gratings follow the same mathematical relation used by Young, albeit with more intense constructive interference fringes than for two slits. For diffraction gratings with 500 lines $/ \mathrm{mm}$ or greater, two issues arise because of small slit spacing.

First, because of the large angle required to satisfy Young's relation for constructive interference, very few interference fringes are observed and can be measured. This can result in students analyzing interference data within the framework of point-by-point calculations instead of relying on the power of graphical analyses to determine wavelength $\lambda$ or slit spacing $d$. With so few data available, both accuracy and precision of final results may suffer.

Second, this author has consistently noted that the laboratory students will often rely on the small angle approximation for analyzing all interference data. While this issue may depend on how Young's relation is derived in the lecture portion of a course or which text is used, many students utilize the small angle version of Young's relation without understanding the conditions when the approximation is valid. For gratings with 500 lines/mm or greater, the small angle approximation is invalid, resulting in significant deviations from expected wavelengths or slit spacing.

To address these issues, a plot of the distance from the central maximum to interference fringes on either side $(x)$ versus the distance from the grating to the central maximum of the interference pattern $(L)$ can be generated. The resulting graph is linear, as will be shown and discussed in the following sections. This graphical technique was first utilized Spring 2018 in a sophomore/junior level laboratory course as presented in the next section.

\section{BACKGROUND AND METHODS}

Physics majors at Appalachian State University are required to take a data analysis laboratory course ("Intermediate Lab") during their sophomore or junior year, prior to taking the capstone laboratory course. Before performing any laboratory activities in multi-slit interference, students derived Young's relation, $d \sin \theta=n \lambda$ [1-2], where $d$ is the spacing between adjacent slits, $\theta$ is the angle from the central maximum, $\lambda$ is the wavelength of light, and $n$ is the order of the interference fringe to either side of the central maximum. Figure 1 shows the geometry of multi-slit interference with key dimensions indicated in the diagram. In determining $\theta$, the distance between the central maximum and the $n^{\text {th }}$ fringe, $x_{n}$, and the distance from the grating to the central maximum, $L$, are measured.

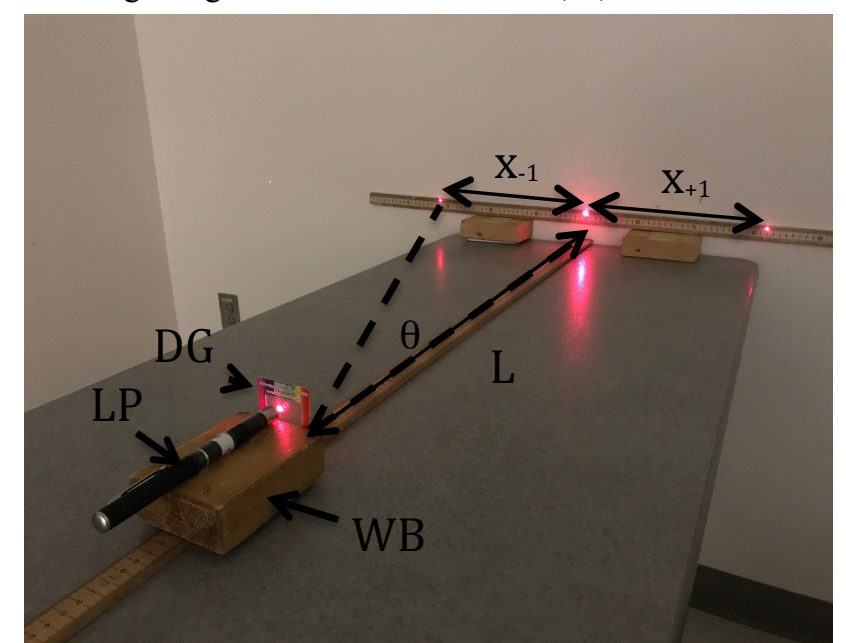

FIG 1. Picture of multi-slit interference set-up used by students. A "red" laser pointer (LP) shines light through a 500 lines/mm diffraction grating (DG) which is located a distance $L$ from where the central maximum fringe forms. Both the pointer and grating are taped to a wooden block (WB) free to move along a 2-meter stick. Distances $x_{-1}$ and 
$x_{+1}$ indicate first order fringes as measured from the central maximum. The angle $\theta$ is between the two dashed lines.

Based on their Young's relation derivation, students were given a sample data set of $x$ and $L$ for $n=1$ fringes to plot and analyze to obtain a value for $\lambda$. Of the nineteen students completing the assignment, sixteen obtained incorrect values for $\lambda$ because the small-angle approximation was incorrectly applied to the situation. The remaining three students obtained the correct wavelength, but only through calculation instead of graphical analysis. Both situations supported the author's prior experience with student approaches to data analysis, as described in the Introduction.

Students were reassigned the graphing activity until it was successfully completed, including error propagation for the wavelength. Students were also tasked with collecting data to obtain the wavelength of two different Laserlands ${ }^{\mathrm{TM}}$ laser pointers [3] utilizing a set-up similar to the one shown in Fig. 1. A 500 lines/mm grating from Rainbow Symphony [4] was taped to the end of a wooden block that was placed on top of a 2-meter stick to record $L$. The laser pointer's "On" button was taped so the laser remained on during the experiment. The laser was taped atop the wooden block with light passing through the grating to generate interference fringes equidistant from the central maximum. A 1-meter stick was used to measure $x$ on either side of the central maximum to generate $x_{a v g}$. An example of the data analysis process is outlined, complete with sample data obtained by the author for a "red" laser, in the following section.

\section{DATA ANALYSIS PROCESS}

The key to correctly analyzing average fringe-to-central maximum distance $x_{\text {avg }}$ versus grating-to-central maximum distance $L$ is utilizing the geometry represented in Fig. 1 and replacing $\sin \theta$ with $\sin \theta=x_{\text {avg }} / \sqrt{L^{2}+x_{a v g}^{2}}$. For the first order situation $(n=1)$, Young's relation can algebraically be rearranged to yield

$$
x_{a v g}=\left(\left(\frac{d}{\lambda}\right)^{2}-1\right)^{-\frac{1}{2}} L
$$

where the term in front of $L$ is the slope. Data were graphed using OriginPro 8.6 [6], with Origin generating the linear regression fit. The plot from an example data set using a "red" laser pointer can be seen in Fig. 2 with the equation generated by Origin as $x_{\text {avg }}=(0.3445 \pm 0.0005) L+(-0.03$ $\pm 0.06 \mathrm{~cm}$ ). The uncertainties in the slope and intercept were generated by Origin using a least squares analysis. It is important to note the intercept should be zero (refer to Eq. 1) and is essentially zero when the uncertainty is taken into account.

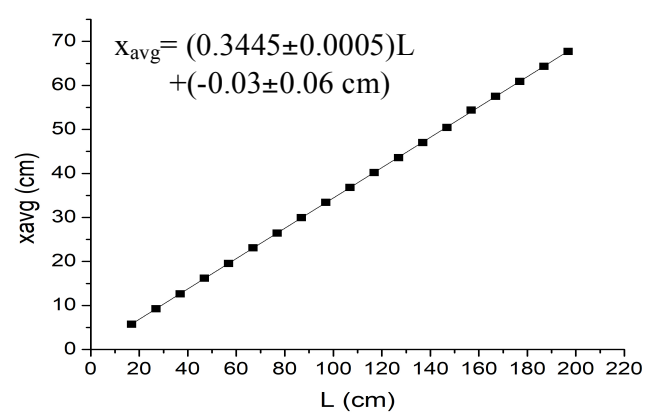

FIG 2. Multi-slit interference data from a "red" laser pointer and a 500 lines/mm grating plotted using Origin [5]. A linear regression fit was applied, as shown by the solid line, with the equation shown above. Error bars were not included for this plot.

To obtain the wavelength, Eq. 1 was algebraically rearranged to obtain

$$
\lambda=d\left(\frac{1}{\text { slope }^{2}}+1\right)^{-\frac{1}{2}}
$$

resulting in a value of $650.5 \mathrm{~nm}$ for the red laser when the slope value of 0.3445 and $d=(1 \mathrm{~mm} / 500$ lines $)=2000 \mathrm{~nm}$ are used. When the general form for error propagation [6] is applied to Eq. 2, the uncertainty in the wavelength $\delta \lambda$ can be found using

$$
\delta \lambda=\frac{d \delta \text { slope }}{\left(1+\text { slope }^{2}\right)^{3 / 2}}
$$

where Sslope is the Origin-generated uncertainty in the slope, and the uncertainty in grating spacing $d$ is assumed to be negligible. [7] For the example data set, $\delta \lambda=0.9 \mathrm{~nm}$, 
resulting in a final value for the wavelength of $650.5 \pm 0.9$ nm.

\section{DISCUSSION}

When students incorrectly applied the small angle approximation to the example data set, the slope was related to $\lambda / d$, resulting in a wavelength of $689.0 \pm 1.0 \mathrm{~nm}$. (Error propagation calculations were used to obtain the uncertainty via $\delta \lambda=d(\delta$ slope $)$.) Since the Laserlands ${ }^{\mathrm{TM}}$ website indicates a value of $650 \mathrm{~nm}$ for their red lasers, the small angle approximation resulted in a $6 \%$ error, compared to the $0.23 \%$ error found by correctly analyzing the data. In addition, the precision was improved slightly by avoiding the small angle approximation.

Actual results from student experiments were less accurate and precise than the example data set collected by the author. Even when students had plenty of space, $L$ was varied over short distances $(<50 \mathrm{~cm})$ resulting in high fractional uncertainties $\left(\delta x_{a v g} / x_{a v g}\right.$ and $\left.\delta L / L\right)$ leading to plots with more scatter than that shown in Fig. 2. Despite that restriction, students were still able to consistently obtain wavelengths with less than $2 \%$ error from Laserlands ${ }^{\mathrm{TM}}$ reported values for violet, blue, green, orange-red, and red lasers, with uncertainties of $5 \mathrm{~nm}$ or less. Even though the bandwidth for the Laserlands ${ }^{\mathrm{TM}}$ laser pointers is not specified, the peak wavelength for the lasers can still be obtained.

The biggest issues in utilizing this graphical approach are student algebraic and graphical analysis skills. Most of the sophomore/junior physics majors taking Intermediate Lab required multiple attempts before obtaining Eq. 1 due to basic algebraic issues like combining terms and squaring all items in the equation. The only guidance instructors provided was to remind intermediate students to use trigonometry to eliminate $\sin \theta$. Lower level students may require instructor guidance to see how Young's relation and Eq. 1 are related. One suggestion for introductory instructors is to provide waypoints for what students can expect to find at different stages of the derivation.

Interpreting the graph was far more challenging to intermediate students than the algebra. Students continued to apply $y=m x+b$ despite prior graphing exercises in the course. To remedy this issue, examples of linear graphs were discussed and connected, variable by variable, to the generic math relation for straight lines. Most examples were taken from introductory lab activities, such as writing equations for velocity versus time and net force versus acceleration for a cart pulled along a track by a hanging mass.

Students were also unaccustomed to seeing the slope as a combination of constants. An example that helped most students was the curve-fitting experience during the freefall experiment where the curve fit equation of $y=A x^{2}+B t$ $+C$ was related to $y=1 / 2 a t^{2}+v_{i} t+y_{i}$, as long as $A=1 / 2 a, B$ $=v_{i}$, and $C=y_{i}$. Seeing that the acceleration was twice the curve-fit constant, $A$, helped students realize the slope has to be interpreted for each situation.

The author spent time with each student showing how Eq. 1 related to the slope and how to interpret linear graphs until each student was able to correctly analyze both the example data set and their own data. The above solutions were sufficient for most students to complete data analysis for grating data.

While the time demands to help students correctly analyze multi-slit interference data using $x_{a v g}$ versus $L$ were greater than anticipated, there were some long-term benefits. Student learned to avoid misuse of the small angle approximation and not to follow text or online shortcuts without understanding when they are valid. Subsequent graphical analyses, either linear or non-linear, during Intermediate $\mathrm{Lab}$ and the capstone course required less instructor assistance than prior offerings of the courses. In addition, students were more likely to consider graphical analysis before using an equation to analyze data than in the past.

\section{CONCLUSION}

Multi-slit interference data, especially for gratings with 500 lines/mm or larger, can be graphically analyzed by measuring constructive interference fringe to central maximum distance as a function of grating to central maximum distance. The slope of the resultant graph is indirectly related to wavelength and slit spacing, either of which can be obtained through algebra. Wavelength results for laser pointers can be obtained with high accuracy $(<2 \%)$ and high precision $(<1 \%)$ for most student experiments. In addition, this approach can help students in upper-division courses with interpretation of curve-fit equations, especially when curve-fit constants are indirectly related to physical quantities of interest. 


\section{ACKNOWLEDGEMENTS}

The author wishes to thank Dr. Scott Thomas for coteaching the data analysis lab course and all the students from Spring 2018 Intermediate Lab, sections 201 to 203, who helped to troubleshoot the first offering of the Optics unit. Thanks also to Dr. Brooke Hester for her feedback on Optics content.
[1] The derivation for Young's relation was not included in this paper as it can be found online (http://web.mit.edu/viz/EM/visualizations/coursenotes/ modules/guide14.pdf. Retrieved 1/5/2018) and in introductory physics texts like the one in Reference 2.

[2] D. C. Giancoli, Physics: Principles with Applications, $7^{\text {th }} E d$. (Prentice Hall, New York, 2014), p. 683.

[3] Laserlands ${ }^{\mathrm{TM}}$ website: https://www.laserlands.net/laser-pointer.html.

[4] Rainbow Symphony, Inc, Reseda, CA 91335, website: www.rainbowsymphony.com .
[5] OriginLab Corporation, Northhampton, MA 01060, website: www.originlab.com .

[6] J.R. Taylor, An Introduction to Error Analysis: The Study of Uncertainties in Physical Measurements, $2^{\text {nd }}$ $E d$. (University Science Books, Sausalito, CA, 1997), p. 75 .

[7] The Rainbow Symphony gratings used in this study were tested and found to have uncertainties of less than 1 line $/ \mathrm{mm}$ for both the 500 lines $/ \mathrm{mm}$ and 1000 lines/mm gratings. 